UCRHEP-T198

July 1997

\title{
TWO HIGGS DOUBLETS MODELS AND CP VIOLATING HIGGS EXCHANGE IN $e^{+} e^{-} \rightarrow t \bar{t} Z$
}

\author{
S. Bar-Shalom ${ }^{a}$, D. Atwood ${ }^{b}$ and A. Soni ${ }^{c}$
}

${ }^{a}$ Physics Dept., University of California, Riverside CA 92521, USA.

${ }^{b}$ Theory Group, Thomas Jefferson National Accelerator Facility, Newport News, VA 23606, USA.

${ }^{c}$ Physics Dept., Brookhaven Nat. Lab., Upton NY 11973, USA.

\begin{abstract}
Appreciable CP Asymmetries $(\sim 10 \%)$ can arise in the reaction $e^{+} e^{-} \rightarrow t \bar{t} Z$ already at tree-level in models with two Higgs doublets. For a neutral Higgs particle, $h$, with a mass in the range 50 $\mathrm{GeV} \lesssim m_{h} \lesssim 400 \mathrm{GeV}$, it may be possible to detect a $2-3$ sigma CP-odd effect in $e^{+} e^{-} \rightarrow t \bar{t} Z$ in $\sim 1-2$ years of running of a future high energy $e^{+} e^{-}$collider with c.m. energies of $\sim 1-2 \mathrm{TeV}$ and an integrated luminosity of 200-500 inverse fb.
\end{abstract}


A future high energy $e^{+} e^{-}$collider running at c.m. energies of $0.5-2 \mathrm{TeV}$, often referred to as the Next Linear Collider (NLC), will no doubt serve as a very useful laboratory for a detailed study of the properties of the Higgs particle(s) and that of the top quark [1]. In particular, it may unveil new phenomena, beyond the Standard Model (SM) associated with the top Yukawa couplings to scalar particle(s). Evidence of such new $t t H$ couplings, if detected at the NLC, can give us important clues about the nature of the scalar potential and of the properties of the scalar particle(s).

In the SM, the scalar potential is economically composed of only one scalar doublet. Even a mild extension of the SM with an additional scalar doublet [2], can give rise to rich new phenomena beyond the SM associated with top-Higgs systems, e.g., tree-level CP-violation [3, 5] and tree-level flavorchanging-scalar (FCS) transitions [6], in interaction of neutral scalars with the top quark. Indeed, the top quark, being so heavy, $m_{t} \sim 175 \mathrm{GeV}$, is the most sensitive to these new interactions.

In this Letter we explore the possibility of detecting tree-level $\mathrm{CP}$-violation in the reaction $e^{+} e^{-} \rightarrow$ $t \bar{t} Z$. We find that in the best cases one needs about one thousand $t \bar{t} Z$ events to observe a 3-sigma CP-nonconserving signal, which, as we will show here, may well be within the experimental reach of the NLC. To some extent, our findings for $e^{+} e^{-} \rightarrow t \bar{t} Z$ are somewhat similar to our previous study of $e^{+} e^{-} \rightarrow t \bar{t} h$ where large tree-level $\mathrm{CP}$ violation was reported [0] . The process $e^{+} e^{-} \rightarrow t \bar{t} Z$ provides another independent, but analogously, promising venue to search for the signatures of the same CP-odd phase, residing in the top-neutral Higgs coupling, in future experiments.

In the presence of two Higgs doublets the most general Yukawa lagrangian can be written as:

$$
\mathcal{L}_{Y}=U_{i j}^{1} \bar{q}_{i, L} \tilde{\phi}_{1} u_{j, R}+D_{i j}^{1} \bar{q}_{i, L} \phi_{1} d_{j, R}+U_{i j}^{2} \bar{q}_{i, L} \tilde{\phi}_{2} u_{j, R}+D_{i j}^{2} \bar{q}_{i, L} \phi_{2} d_{j, R}+\text { h.c. }
$$

where $\phi_{i}$ for $i=1,2$ are the two scalar doublets and $U_{i j}^{k}, D_{i j}^{k}$, for $k=1,2$, are the Yukawa couplings matrices which are in general non-diagonal. Depending on the assumptions made, one can then obtain different versions of a Two Higgs Doublet Model (2HDM). In particular, if one imposes the discrete symmetries $\phi_{1} ; \phi_{2} \rightarrow-\phi_{1} ; \phi_{2}$ and $d_{i, R} ; u_{i, R} \rightarrow-d_{i, R} ;-u_{i, R}$ or $-d_{i, R} ; u_{i, R}$ one arrives at the so called Model I or Model II, respectively, depending on whether the $-1 / 3$ and $2 / 3$ charged quarks are coupled to the same or to different scalar doublets. If, in addition, these discrete symmetries are softly violated by a mass-dimension-two term in the Higgs potential, then the real and imaginary parts of the Higgs doublets mix, giving rise to CP-violating scalar-pseudoscalar mixed couplings of a neutral Higgs to fermions already at the tree-level [7]. On the other hand, if one does not impose the above discrete symmetries, one arrives at a most general version of the 2HDM, often called 
Model III, in which both FCS transitions and CP-nonconserving interactions between the neutral Higgs particles and fermions are present at tree-level (see e.g., Luke and Savage in [6] and [8]).

The scalar spectrum of any of the above 2HDM's consists of three neutral Higgs and two charged Higgs particles. The $\mathcal{H}^{k} q \bar{q}$ and $\mathcal{H}^{k} Z Z(k=1,2,3$ corresponding to the three neutral Higgs particles $\mathcal{H}^{k}$ and $q$ stands for quark) interaction lagrangian parts of a general 2HDM can be written as:

$$
\mathcal{L}_{\mathcal{H}^{k} q q}=-\frac{g_{W}}{\sqrt{2}} \frac{m_{q}}{m_{W}} \mathcal{H}^{k} \bar{q}\left(a_{q}^{k}+i b_{q}^{k} \gamma_{5}\right) q \quad, \quad \mathcal{L}_{\mathcal{H}^{k} Z Z}=\frac{g_{W}}{c_{W}} m_{Z} c^{k} \mathcal{H}^{k} g_{\mu \nu} Z^{\mu} Z^{\nu}
$$

Note that in the SM the couplings in Eq. 2, of the only neutral Higgs present, are $a_{q}=1 / \sqrt{2}, b_{q}=0$ and $c=1$ and there is no phase in the $\mathcal{H}^{k} q \bar{q}$ coupling. In Model II, for up quarks for example [0]:

$$
a_{u}^{k}=R_{1 k} / \sin \beta, \quad b_{u}^{k}=R_{3 k} / \tan \beta, \quad c^{k}=R_{1 k} \sin \beta+R_{2 k} \cos \beta,
$$

where $\tan \beta \equiv v_{u} / v_{d}$ and $v_{u}\left(v_{d}\right)$ is the vacuum-expectation-value responsible for giving mass to the up(down) quark. $R$ is the neutral Higgs mixing matrix which can be parameterized by three Euler angles $\alpha_{1,2,3}$ [7].

A general feature of the above 2HDM's is that only two out of the three neutral Higgs can simultaneously have a coupling to vector bosons and a pseudoscalar coupling to fermions. We will denote these two neutral Higgs by $h$ and $H$ with couplings $a_{q}^{h}, b_{q}^{h}, c^{h}$ and $a_{q}^{H}, b_{q}^{H}, c^{H}$, corresponding to the light and heavy neutral Higgs, respectively.' Then, an important aspect of these 2HDM's, which has crucial phenomenological implications for CP-violation, is that these couplings are subject to the constraint $b_{q}^{h} c^{h}+b_{q}^{H} c^{H}=0$ [5]. This implies the existence of a "GIM"-like cancellation, namely; all $\mathrm{CP}$-violating effects due to the Higgs sector, being proportional to $b^{h} c^{h}+b^{H} c^{H}$, must vanish when the two Higgs states $h$ and $H$ are degenerate.

We now discuss the possibility of having CP-violation, already at the tree-level, driven by 2HDM's, in our reaction:

$$
e^{+}\left(p_{+}\right)+e^{-}\left(p_{-}\right) \rightarrow q\left(p_{q}\right)+\bar{q}\left(p_{\bar{q}}\right)+Z\left(p_{Z}\right)
$$

In the unitary gauge the reaction in Eq. H can proceed via the Feynman diagrams depicted in Fig. 1. Diagram $b$, where a pair of $Z \mathcal{H}$ is produced $\left(\mathcal{H}\right.$ is produced either as real or virtual, i.e. $m_{\mathcal{H}}>2 m_{t}$ or $m_{\mathcal{H}}<2 m_{t}$ respectively) followed by $\mathcal{H} \rightarrow t \bar{t}$, is the only one where new CP-nonconserving dynamics

\footnotetext{
${ }^{1}$ In some instances we will denote these two neutral Higgs by $\mathcal{H}$. Then $\mathcal{H}=h$ or $H$ is to be understood.
} 
from the Higgs sector can arise being proportional to the CP-odd phase in the $\mathcal{H} q \bar{q}$ vertex. In particular, all CP-violating effects arise from the interference of diagram $b$ with the diagrams of class $a$ in Fig. 1 and are proportional to the quantity $b_{q}^{\mathcal{H}} \times c^{\mathcal{H}}$.

A detailed cross-section analysis of the reaction $e^{+} e^{-} \rightarrow t \bar{t} Z$ was performed in the SM by Hagiwara et al. in [9]. There, it was found that the Higgs exchange contribution of diagram $b$ in Fig. 1 will be almost invisible in a $\mathrm{TeV} e^{+} e^{-}$collider for neutral Higgs masses in the range $m_{h}<2 m_{t}$. On the contrary, we will show here that if the scalar sector is doubled, then the lightest neutral Higgs may reveal itself through CP-violating interactions with the top quark even if $m_{h}<2 m_{t}$. We will sketch below the main characteristics of the total differential cross-section (DCS) and focus primarily on its CP-violating part. The tree-level polarized DCS, $\Sigma_{(j)}^{0}, j=-1(1)$ for left(right) handed electrons, is in general a sum of two terms: the CP-even and odd terms $\Sigma_{+(j)}^{0}$ and $\Sigma_{-(j)}^{0}$, respectively, i.e. $\Sigma_{(j)}^{0} \equiv \Sigma_{+(j)}^{0}+\Sigma_{-(j)}^{0}$. However, we can furthermore divide $\Sigma_{ \pm(j)}^{0}$ into:

$$
\begin{aligned}
& \Sigma_{+(j)}^{0} \equiv \Sigma_{++(j)}^{0(S M)}+\Sigma_{++(j)}^{0(\mathcal{H})}+\Sigma_{+-(j)}^{0(\mathcal{H})}, \\
& \Sigma_{-(j)}^{0} \equiv \Sigma_{-+(j)}^{0(\mathcal{H})}+\Sigma_{--(j)}^{0(\mathcal{H})},
\end{aligned}
$$

where the first and second subscripts denote the CP property and the $T_{N}$ property ( $T_{N}$ is the naive time reversal operator defined by replacing time with its negative without switching initial and final states) of the DCS's in Eqs. 5 and 6, respectively. The superscript indicates if it is a pure SM contribution, coming from diagrams $a$ and denoted by (SM), or interference terms associated with diagram $b$ and denoted by $(\mathcal{H})$. Thus, for example, $\Sigma_{--(j)}^{0(\mathcal{H})}$ is the CP-odd, $T_{N^{-o d d}}$ polarized DCS, upon which we will concentrate, that emanates from the interference of diagram $b$ with the SM diagrams $a$ in Fig. 1.

It is then very simple to identify each of the DCS's in Eqs. 5 and 6 associated with the 2HDM-SM and the 2HDM-2HDM interferences in terms of the Higgs coupling constants $a_{q}^{\mathcal{H}}, b_{q}^{\mathcal{H}}$ and $c^{\mathcal{H}}$ defined in Eq. 2. In particular we find:

$$
\begin{aligned}
\Sigma_{++(j)}^{0(\mathcal{H})}= & a_{q}^{h} c^{h} \operatorname{Re}\left(\Pi_{h}\right) f_{++(j)}^{1}+\left(a_{q}^{h} c^{h}\right)^{2}\left(\operatorname{Re}\left(\Pi_{h}\right) f_{++(j)}^{2}+\operatorname{Im}\left(\Pi_{h}\right) f_{++(j)}^{3}\right) \\
& +\left(b_{q}^{h} c^{h}\right)^{2}\left(\operatorname{Re}\left(\Pi_{h}\right) f_{++(j)}^{4}+\operatorname{Im}\left(\Pi_{h}\right) f_{++(j)}^{5}\right)+(h \rightarrow H), \\
\Sigma_{+-(j)}^{0(\mathcal{H})}= & a_{q}^{h} c^{h} \operatorname{Im}\left(\Pi_{h}\right) f_{+-(j)}^{1}+(h \rightarrow H),
\end{aligned}
$$




$$
\begin{aligned}
& \Sigma_{-+(j)}^{0(\mathcal{H})}=b_{q}^{h} c^{h} \operatorname{Im}\left(\Pi_{h}\right) f_{-+(j)}^{1}+(h \rightarrow H), \\
& \Sigma_{--(j)}^{0(\mathcal{H})}=b_{q}^{h} c^{h} \operatorname{Re}\left(\Pi_{h}\right) f_{--(j)}^{1}+(h \rightarrow H),
\end{aligned}
$$

where:

$$
\Pi_{\mathcal{H}} \equiv\left(s+m_{Z}^{2}-m_{\mathcal{H}}^{2}-2 p \cdot p_{Z}+i m_{\mathcal{H}} \Gamma_{\mathcal{H}}\right)^{-1} .
$$

$p \equiv p_{-}+p_{+}$and $\Gamma_{\mathcal{H}}$ is the width of $\mathcal{H} . f_{m n(j)}^{\ell}, m, n=+$ or - , are kinematical functions of phase space which transform like $m$ under $\mathrm{CP}$ and like $n$ under $T_{N}$. A few important remarks are in order at this stage:

1. The functions $f_{--(j)}^{1}$ and $f_{+-(j)}^{1}$, being $T_{N^{-o d d}}$, are proportional to the Levi-Civita tensor $\epsilon\left(p_{-}, p_{+}, p_{q}, p_{\bar{q}}\right)$.

2. There is no term proportional to $a_{q}^{\mathcal{H}} b_{q}^{\mathcal{H}}$ in the DCS's at tree-level.

3. The diagrams where the $Z$ is emitted from the incoming electron and positron lines, do not contribute to $\Sigma_{--(j)}^{0(\mathcal{H})}$.

4. $\Sigma_{+-(j)}^{0(\mathcal{H})}$ and $\Sigma_{-+(j)}^{0(\mathcal{H})}$ are proportional to the absorptive part coming from the Higgs propagator, $\operatorname{Im}\left(\Pi_{h}\right)$, and are therefore not pure tree-level quantities being proportional to the Higgs width. Thus, a consistent calculation of the +- and -+ parts of the DCS has to include the full next order (i.e., 1-loop order) contribution in perturbation theory. In contrast, $\Sigma_{--(j)}^{0(\mathcal{H})}$, being an odd function of $T_{N}$, is proportional to $\operatorname{Re}\left(\Pi_{h}\right)$ and is therefore a pure tree-level quantity.

We will concentrate here on the CP-odd $T_{N^{-}}$odd effects emanating from $\Sigma_{--(j)}^{0(\mathcal{H})}$ which, as mentioned above, is proportional at the tree-level to the interference of the SM-like diagrams where the $Z$ is radiated off the $t$ or $\bar{t}$ with the Higgs exchange diagram [10]. By measuring a CP-odd $T_{N}$-odd observable one can extract information on the magnitude of $b_{q}^{\mathcal{H}} c^{\mathcal{H}}$. However, note that the full DCS contains more information about the other scalar coupling combinations. Thus with the appropriate optimal observables [11], it is possible to isolate each coupling combination in Eqs. 0-10, and therefore, in principle, to identify the exact quantum numbers of the neutral Higgs particle, say the lightest one, which is exchanged in diagram $b$. This technique was applied to the reaction $e^{-} e^{+} \rightarrow t \bar{t} h$ by Gunion et al. in $\llbracket$. However, we expect that the reaction $e^{-} e^{+} \rightarrow t \bar{t} Z$ will be less sensitive to the CP-even top-Higgs couplings due to the dominating presence of the SM diagrams a depicted in Fig. 1.

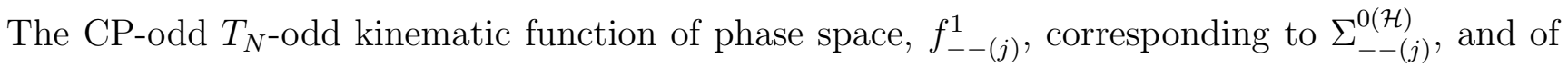
relevance to the present analysis is: 


$$
\begin{aligned}
f_{--(j)}^{1}= & -\sqrt{2}\left(\frac{2 g_{W}^{3}}{c_{W}^{3}}\right)^{2} \frac{m_{q}^{2}}{m_{Z}^{2}} \Pi_{Z} T_{q}^{3} c_{j}^{Z} \epsilon\left(p_{-}, p_{+}, p_{q}, p_{\bar{q}}\right) \times \\
& \left\{j\left(\Pi_{q}+\Pi_{\bar{q}}\right)\left[m_{Z}^{2} w_{j}^{-}+\left(s_{t}-s\right) w_{j}^{+}\right]+T_{q}^{3} c_{j}^{Z} \Pi_{Z}\left(\Pi_{q}-\Pi_{\bar{q}}\right) f\right\},
\end{aligned}
$$

where:

$$
w_{j}^{ \pm} \equiv\left(s_{W}^{2} Q_{q}-\frac{1}{2} T_{q}^{3}\right) c_{j}^{Z} \Pi_{Z} \pm Q_{q} s_{W}^{2} c_{W}^{2} \Pi_{\gamma}
$$

Here $c_{-1}^{Z}=1 / 2-s_{W}^{2}, c_{1}^{Z}=-s_{W}^{2}$ (recall that $j=-1(1)$ for a left(right) handed electron). $s_{W}\left(c_{W}\right)$ is the $\sin (\cos )$ of the weak mixing angle $\theta_{W}$ and $Q_{q}$ and $T_{q}^{3}$ are the charge and $z$-component of the weak isospin of the quark, respectively. Furthermore:

$$
\Pi_{Z} \equiv\left(s-m_{Z}^{2}\right)^{-1} \quad, \quad \Pi_{\gamma} \equiv s^{-1}, \Pi_{q(\bar{q})} \equiv\left(m_{Z}^{2}+2 p_{q(\bar{q})} \cdot p_{Z}\right)^{-1}
$$

where $s=\left(p_{-}+p_{+}\right)^{2}$ is the c.m. energy squared of the colliding electrons, $s_{t} \equiv\left(p_{q}+p_{\bar{q}}\right)^{2}$ and we have also defined the CP-odd quantity $f \equiv\left(p_{-}-p_{+}\right) \cdot\left(p_{q}+p_{\bar{q}}\right)$.

As mentioned earlier, being an odd function of the $T_{N}$ symmetry operation, $f_{--(j)}^{1}$ can only probe CP-asymmetries of the $T_{N^{-}}$odd type in $e^{-} e^{+} \rightarrow t \bar{t} Z$. This leads us to consider the following dimensionless CP-odd, $T_{N}$-odd observables:

$$
O=\frac{\vec{p}_{-} \cdot\left(\vec{p}_{q} \times \vec{p}_{\bar{q}}\right)}{s^{3 / 2}}, \quad O_{\mathrm{opt}}=\frac{\Sigma_{--}^{0(\mathcal{H})}}{\Sigma_{+}^{0}} .
$$

$O_{\text {opt }}$ is an optimal observable in the sense that the statistical error, in the measured asymmetry, is minimized [11]. Also, note that both observables are proportional to $\epsilon\left(p_{-}, p_{+}, p_{q}, p_{\bar{q}}\right)$ since there is only one possible independent triple correlation product (or equivalently a Levi-Civita tensor) when the final state consists of three particles only and the spins are disregarded. In particular, $O_{\text {opt }}$ is related to $O$ by a multiplication by a CP-even function. The theoretical statistical significance, $N_{S D}$, in which an asymmetry can be measured in an ideal experiment is given by $N_{S D}=A \sqrt{L} \sqrt{\sigma_{t t Z}}$ where for the observables $O$ and $O_{\text {opt }}$ the asymmetry $A$, defined above, is:

$$
A_{O} \approx\langle O\rangle / \sqrt{\left\langle O^{2}\right\rangle}, A_{\mathrm{opt}} \approx \sqrt{\left\langle O_{\mathrm{opt}}\right\rangle}
$$


Also, $\sigma_{t t Z} \equiv \sigma\left(e^{-} e^{+} \rightarrow t \bar{t} Z\right)$ is the cross-section and $L$ is the effective luminosity for fully reconstructed $t \bar{t} Z$ events. In particular, we will take $L=\epsilon \mathcal{L}$, where $\mathcal{L}$ is the total yearly integrated luminosity $]$ and $\epsilon$ is the overall efficiency for reconstruction of the $t \bar{t} Z$ final state. Note that detection of the asymmetries in Eq. 16 requires the identification of the $t$ and $\bar{t}$ as well as the reconstruction of their momenta. Thus, the most suitable scenario is when either the $t$ or the $\bar{t}$ decays semi-leptonically and the other decays hadronically. Distinguishing between $t$ and $\bar{t}$ in the double hadronic decay case will require more effort and still remains an experimental challenge.

In Fig. 2 we plot the cross-section, $\sigma_{t t Z}^{I I}$ as a function of $m_{h}$ and $\sqrt{s}$, for Model II with $\left\{\tan \beta, \alpha_{1}, \alpha_{2}, \alpha_{3}\right\}=$ $\{0.3, \pi / 2, \pi / 4,0\}$ which we denote as set II. We will adopt set II later also when discussing the CPviolating effect. Here we have set the mass of the heavier Higgs to be $m_{H}=750 \mathrm{GeV}$. We see that $\sigma_{t t Z}^{I I}$ is typically $\sim$ few fb for c.m. energies of $\sim 1-2 \mathrm{TeV}$; it peaks for $m_{h} \approx 2 m_{t}$ and $\sqrt{s} \sim 800$ $\mathrm{GeV}$ at around $7 \mathrm{fb}$. Therefore, with $\mathcal{L} \gtrsim 10^{2}[\mathrm{fb}]^{-1}$ it may be possible to produce $10^{2}-10^{3} t \bar{t} Z$ 's at the NLC running with c.m. energies $\gtrsim 1 \mathrm{TeV}$ scale. We also remark that, although the crosssections in the SM and Model II are of the same order, for certain values of $\left\{\tan \beta, \alpha_{1}, \alpha_{2}, \alpha_{3}\right\}$ and the neutral Higgs mass, there can be a significant difference between the cross-sections predicted by the two-models. For example, with unpolarized incoming electrons and for $\sqrt{s}=1 \mathrm{TeV}, m_{h}=360$ $\mathrm{GeV}$ and $\left\{\tan \beta, \alpha_{1}, \alpha_{2}, \alpha_{3}\right\}=\{0.3, \pi / 2, \pi / 4,0\}, \sigma_{t t Z}^{I I} \simeq 6 \mathrm{fb}$, while the $\mathrm{SM}$ cross-section is $\sigma_{t t Z}^{\mathrm{SM}} \simeq 3.5$ $\mathrm{fb}$. The combined information from a study of the cross-section itself along with CP-violation may be extremely useful in understanding the dynamics of the reaction $e^{+} e^{-} \rightarrow t \bar{t} Z$ although we choose not to pursue in that direction in this paper.

Let us first consider an unpolarized incoming electron beam and concentrate on the CP-odd effect associated with $O_{\text {opt }}$. The effect of the simple triple product $O$ is slightly smaller. In Fig. 3 we present our main results for the expected asymmetry and statistical significance corresponding to $O_{\text {opt }}$ in Model II, as a function of the mass $\left(m_{h}\right)$ of the light Higgs where, again, $m_{H}=750$ $\mathrm{GeV}$. We plot $N_{S D} / \sqrt{L}$, thus scaling out the luminosity factor from the theoretical prediction and as an illustration, for the free parameters of Model II, we adopt set II defined above, i.e. $\left\{\tan \beta, \alpha_{1}, \alpha_{2}, \alpha_{3}\right\}=\{0.3, \pi / 2, \pi / 4,0\}$. We remark that the effect is practically insensitive to $\alpha_{3}$, and $\alpha_{1}=\pi / 2, \alpha_{2}=\pi / 4$ correspond to the best effect, though not unique. Also, the CP-violating effect is roughly proportional to $1 / \tan \beta$, it therefore drops as $\tan \beta$ is increased. However, we find that we can still have $N_{S D} / \sqrt{L}>0.1$ even in the unpolarized case for $\tan \beta \lesssim 0.6, \alpha_{1}=\pi / 2, \alpha_{2}=\pi / 4, \alpha_{3}=0$.

\footnotetext{
${ }^{2}$ For illustrative purposes, we will choose: $\mathcal{L}=200[\mathrm{fb}]^{-1}$ for $\sqrt{s}=1 \mathrm{TeV}$ and $\mathcal{L}=500[\mathrm{fb}]^{-1}$ for $\sqrt{s}=1.5 \mathrm{TeV}$ [1].

${ }^{3}$ Plots of the SM cross-section, $\sigma_{t t Z}^{S M}$, can be found in [9], where it was also found that $\sigma_{t t Z}^{S M} \sim$ few fb.
} 
Evidently the asymmetry is almost insensitive to $m_{h}$ in the range $50 \mathrm{GeV} \lesssim m_{h} \lesssim 2 m_{t}$ where it stays roughly at the $7-8 \%$ level for $\sqrt{s} \sim 1-2 \mathrm{TeV}$ (see Fig. 3 ). In that range $0.1 \lesssim N_{S D} / \sqrt{L} \lesssim 0.2$; it slightly grows as $m_{h}$ is increased and reaches its peak around $m_{h} \approx 2 m_{t}$. For $m_{h}>2 m_{t}$, for which an on-shell $h$ is produced and then decays to a pair of $t \bar{t}$, as $m_{h}$ grows the asymmetry drops till it essentially vanishes when $m_{h} \rightarrow m_{H}$ in which case the "GIM" like cancellation, discussed above, applies. Also, with respect to the c.m. energy, both $A_{\text {opt }}$ and $N_{S D} / \sqrt{L}$ reach their peak values at around $\sqrt{s} \sim 1 \mathrm{TeV}$ for both $m_{h}=100$ and $360 \mathrm{GeV}$ and weakly fall as the c.m. energy is increased. For example, we find that with $m_{h}=100(360) \mathrm{GeV}$ and $\epsilon=0.5$, it may be possible to observe a $\mathrm{CP}$-nonconserving effect in the reaction $e^{-} e^{+} \rightarrow t \bar{t} Z$ with a statistical significance of $N_{S D} \approx 1.6(2.0)$ for $\sqrt{s}=1 \mathrm{TeV}$ and $\mathcal{L}=200[\mathrm{fb}]^{-1}$, and $N_{S D} \approx 2.1(2.5)$ for $\sqrt{s}=1.5 \mathrm{TeV}$ and $\mathcal{L}=500[\mathrm{fb}]^{-1}$ if the incoming electrons are unpolarized.

In Table 1 we present $N_{S D}$ for $O_{\text {opt }}$, in Model II with set II, for polarized and unpolarized electrons. For illustrative purposes, we choose $m_{h}=100,160$ and $360 \mathrm{GeV}$ and, as before, we present the numbers for $\sqrt{s}=1 \mathrm{TeV}$ with $\mathcal{L}=200[\mathrm{fb}]^{-1}$ and for $\sqrt{s}=1.5 \mathrm{TeV}$ with $\mathcal{L}=500$ $[\mathrm{fb}]^{-1}$. In both cases we take $\epsilon=0.5$ assuming that there is no loss of luminosity when the electrons are polarized.f Also, to demonstrate the sensitivity of the CP-effect to the mass $\left(m_{H}\right)$ of the heavy Higgs, we present numbers for both $m_{H}=750 \mathrm{GeV}$ (shown in the parentheses) and $m_{H}=1 \mathrm{TeV}$. We see from Table 1 that left polarized incoming electrons can probe CP-violation slightly better than unpolarized ones. In particular, for $m_{H}=1 \mathrm{TeV}$ we find that with left polarized electrons and for $\sqrt{s}=1(1.5) \mathrm{TeV}$, the CP-effect is above the 2(3)-sigma level for $m_{h} \gtrsim 2 m_{t}$. At c.m. energy $\sqrt{s}=1 \mathrm{TeV}$ the CP-effect is practically insensitive to the choice $m_{H}=750 \mathrm{GeV}$ or $m_{H}=1 \mathrm{TeV}$. However, we see from the Table that as one goes to $\sqrt{s}=1.5 \mathrm{TeV}, m_{H}=1 \mathrm{TeV}$ can give rise to a 3 -sigma signal in the range, $100 \mathrm{GeV} \lesssim m_{h} \lesssim 400 \mathrm{GeV}$, if the electrons are negatively polarized, while with $m_{H}=750 \mathrm{GeV}$ the $\mathrm{CP}$-signal is smaller by about half a sigma. We remark again that the results for the simple observable $O$ exhibit the same behavior though slightly smaller then those for $O_{\mathrm{opt}}$.

Before summarizing we wish to emphasize that the analysis performed here for Model II can be generalized to models I and III as well. Recall that in Model II a mass-dimension-two operator, that softly breaks the discrete symmetry which is responsible for natural-flavor-conservation (NFC), is needed in order to have CP-violati ng Higgs-fermion couplings. On the other hand, in Model III

\footnotetext{
${ }^{4}$ If the efficiency for $t \bar{t} Z$ reconstruction is $\epsilon=0.25$ then our numbers would correspondingly require 2 years of running.
} 
there is no NFC and the CP-odd phase in the $\mathcal{H}^{k} q q$ vertex can arise from a phase in the Yukawa couplings $U_{i j}^{2}$ and $D_{i j}^{2}$ defined in Eq. 1 (for more details see [8]). The pseudoscalar coupling in Eq. 2, responsible for CP-violation, can be chosen (in Model III) as $b_{q}^{k} \propto \lambda_{q}$ [12], where $\lambda_{q}$ is a free parameter of the model expected to be of $\mathcal{O}(1)\left[8\right.$. Then the replacement $\lambda_{t} \approx 1 / \tan \beta$ (for a given value of $\tan \beta$ in Model II) can give rise to comparable CP-nonconserving effects in $e^{+} e^{-} \rightarrow t \bar{t} Z$. Thus, the main difference between Model II and Model III arises from the fact that, while in Model II a small $\tan \beta$ is required in order to enhance the CP-odd effect, in Model III the effect is elevated as $\lambda_{t}$ is correspondingly increased. The same argument holds also for other previously suggested CP studies in top systems where the results obtained for Model II can be generalized to Model III. This seems to indicate that even if such CP-violating effects are found in $e^{-} e^{+} \rightarrow t \bar{t} Z$ or other reactions which involve the top-neutral Higgs CP-phase discussed in this paper, it may not be possible to distinguish Model II from Model III, as both models may have a comparable CP-odd phase in the Higgs sector. In that sense, the best way to proceed for correctly classifying the Higgs sector, is to search for large signatures of FC effects in top quark reactions as well. Model III, with FCS couplings to fermions proportional to the fermion masses involved in the FC vertex [12, may indeed drive such large FC effects in top systems, some of which are $e^{+} e^{-} \rightarrow t \bar{c} ; t \bar{c} \nu_{e} \bar{\nu}_{e} ; t \bar{c} e^{+} e^{-} ; t \bar{c} Z ; t \bar{t} c \bar{c} ; t \bar{c} q \bar{q}$ and were investigated in [6]. Detection or no detection of these FC signatures along with evidence for CPviolation in the Higgs sector in high energy $e^{+} e^{-}$colliders, may well be the only way to experimentally distinguish between scalar dynamics of a Model II or a Model III origin.

To summarize, CP-violation in $e^{-} e^{+} \rightarrow t \bar{t} Z$ at a future high energy $e^{+} e^{-}$collider was studied in the context of 2HDM's. An important property of this reaction is that CP-violation arises already at the tree-level through interference of $Z$ emission from the $t$ or $\bar{t}$ and its emission off a s-channel $Z$ and therefore allowing for a relatively large CP-violating signal. In particular, we found that within a broad range of the lightest Higgs mass, $100 \mathrm{GeV} \lesssim m_{h} \lesssim 400 \mathrm{GeV}$ and with c.m. energies between 1-2 TeV the asymmetry can reach the $\sim 10 \%$ level. The corresponding statistical significance, in which it may be observed, is around 2-3 sigma for unpolarized incoming electrons and, if $m_{h} \gtrsim 2 m_{t}$, it can reach above 3 -sigma for polarized ones and c.m. energy of $\sqrt{s}=1.5 \mathrm{TeV}$. Bearing the expected difficulty of observing the Higgs exchange effect in $e^{+} e^{-} \rightarrow t \bar{t} Z$ in the $\mathrm{SM}$ and with Higgs masses below $2 m_{t}$ [9], it is especially gratifying that the CP-effect is sizable and almost insensitive to $m_{h}$ in the range $50 \mathrm{GeV} \lesssim m_{h} \lesssim 2 m_{t} \mathrm{GeV}$. We therefore encourage a detailed scrutiny of the reaction 
$e^{-} e^{+} \rightarrow t \bar{t} Z$ in the NLC. Such an investigation, especially due to the promising CP-nonconserving effects reported here, may be helpful in unraveling the CP properties of the Higgs sector.

This research was supported in part by the U.S. DOE contract numbers DE-AC02-76CH00016(BNL), DC-AC05-84ER40150(Jefferson Lab) and DE-FG03-94ER40837(UCR).

\section{References}

[1] Proceedings of the Workshop on Physics and Experiments with Linear $e^{+} e^{-}$Colliders, eds. F. Harris S. Olsen, S. Pakvasa and X. Tata, World Scientific, Singapore, 1993; A. Miyamoto and Y. Fujii, ibid, 1996.

[2] For a review see J. Gunion, H. Haber, G. Kane and S. Dawson, "The Higgs Hunter's Guide", (Addision-Wesley, New York, 1990).

[3] S. Bar-Shalom, D. Atwood, G. Eilam, R. Mendel and A. Soni, Phys. Rev. D53, 1162 (1996); see also Gunion at el. in Ref. 4.

[4] J.F. Gunion, B. Grzadkowski and X.-G. He, Phys. Rev. Lett 77, 5172 (1996).

[5] D. Atwood and A. Soni, hep-ph/9607481.

[6] M.J. Savage, Phys. Lett. B266, 135 (1991); W.S. Hou, Phys. Lett. B296, 179 (1992); M. Luke and M.J. Savage, Phys. Lett. B307, 387 (1993); L.J. Hall and S. Weinberg, Phys. Rev. D48, R979 (1993); D. Atwood, L. Reina and A. Soni, Phys. Rev. Lett 75, 3800 (1995); ibid Phys. Rev. D53, 1199 (1996); W.-S. Hou and G.-L. Lin, Phys. Lett. B379, 261 (1996); S. Bar-Shalom, G. Eilam, A. Soni and J. Wudka, hep-ph/9703221 to appear in Phys. Rev. Lett. , July (1997).

[7] C.D. Froggat, R.G. Moorhouse and I.G. Knowles, Nucl. Phys. B386 63 (1992); W. Bernreuther, T. Schröder and T.N. Pham, Phys. Lett. B279, 389 (1992).

[8] For a recent short review of Model III see: D. Atwood, L. Reina and A. Soni, Phys. Rev. D55, 3156 (1997). 
[9] K. Hagiwara, H. Murayama and I. Watanabe, Nucl. Phys. B367 257 (1991); see also K. Fujii in the Proceedings of the 4th KEK Topical Conference on Flavor Physics (1996) and references therein.

[10] We remark that one-loop, CP-odd $T_{N^{-}}$-even, effects in $e^{+} e^{-} \rightarrow Z \mathcal{H} \rightarrow t \bar{t} Z$ were investigated by B. Grzadkowski (Phys. Lett. B338, 71 (1994)). Although asymmetries at the order of 50\% were found there, the SM-like diagrams of class $a$ in Fig. 1 were ignored in that analysis. Since those diagrams make significant contributions to the rate, therefore, (as was also mentioned by the author) such large asymmetries will be difficult to materialize unless one is able to experimentally separate the contribution from the Higgs exchange graphs from the rest of the diagrams which lead to the same final state.

[11] D. Atwood and A. Soni, Phys. Rev. D45, 45 (1992).

[12] T.P. Cheng and M. Sher, Phys. Rev. D35, 3484 (1987); M. Sher and Y. Yuan, Phys. Rev. D44, 1461 (1991). 


\section{Figure Captions}

Fig. 1: Tree-level Feynman diagrams contributing to $e^{+} e^{-} \rightarrow t \bar{t} Z$ in a two Higgs doublet model. Diagram $a$ represents 8 diagrams in which either $Z$ or $\gamma$ are exchanged in the s-channel and the outgoing $Z$ is emitted from $e^{+}, e^{-}, t$ or $\bar{t}$

Fig. 2: The cross section (in [fb]) for the reaction $e^{+} e^{-} \rightarrow t \bar{t} Z$, assuming unpolarized electron and positron beams, for Model II with set II and as a function of $m_{h}$ (solid and dashed lines) and $\sqrt{s}$ (dotted and dotted-dashed lines). Set II means $\left\{\tan \beta, \alpha_{1}, \alpha_{2}, \alpha_{3}\right\} \equiv\{0.3, \pi / 2, \pi / 4,0\}$.

Fig. 3: The asymmetry, $A_{\text {opt }}$, and scaled statistical significance, $N_{S D} / \sqrt{L}$, for the optimal observable $O_{\text {opt }}$ as a function of the light Higgs mass $m_{h}$, for $\sqrt{s}=1 \mathrm{TeV}$ and $1.5 \mathrm{TeV}$. See also caption to Fig. 2. 
Table 1: The statistical significance, $N_{S D}$, in which the CP-nonconserving effects in $e^{+} e^{-} \rightarrow t \bar{t} Z$ can be detected in one year of running of a future high energy collider with either unpolarized or polarized incoming electron beam. We have used a yearly integrated luminosity of $\mathcal{L}=200$ and $500[\mathrm{fb}]^{-1}$ for $\sqrt{s}=1$ and $1.5 \mathrm{TeV}$, respectively, and an efficiency reconstruction factor of $\epsilon=0.5$ for both energies. $N_{S D}$ is given for both $m_{H}=750 \mathrm{GeV}$ (in parentheses) and $m_{H}=1 \mathrm{TeV}$. Recall that $j=1(-1)$ stands for right(left) polarized electrons. Set II means $\left\{\tan \beta, \alpha_{1}, \alpha_{2}, \alpha_{3}\right\} \equiv\{0.3, \pi / 2, \pi / 4,0\}$.

\begin{tabular}{|c|c|c|c|c|}
\hline \multirow{3}{*}{$\begin{array}{r}\sqrt{s} \\
(\mathrm{TeV}) \Downarrow \\
\end{array}$} & \multirow{3}{*}{$(\mathrm{GeV}) \stackrel{j}{\Rightarrow}$} & \multicolumn{3}{|c|}{$e^{+} e^{-} \rightarrow t \bar{t} Z$ (Model II with Set II) } \\
\hline & & \multicolumn{3}{|c|}{$O_{\mathrm{opt}}$} \\
\hline & & $m_{h}=100$ & $m_{h}=160$ & $m_{h}=360$ \\
\hline \multirow{3}{*}{1} & 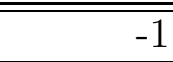 & 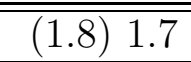 & $\overline{(1.8) 1.8}$ & 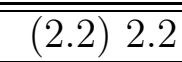 \\
\hline & unpol & (1.6) 1.6 & (1.7) 1.6 & (2.0) 2.0 \\
\hline & 1 & (1.5) 1.5 & (1.5) 1.5 & (1.8) 1.8 \\
\hline \multirow{3}{*}{1.5} & -1 & $\bar{~} \overline{(2.3)} 2.9$ & $\overline{~(2.4) 3.0}$ & $\overline{(2.8) 3.3}$ \\
\hline & unpol & (2.1) 2.6 & (2.1) 2.7 & (2.5) 3.0 \\
\hline & 1 & (1.8) 2.3 & (1.8) 2.3 & (2.1) 2.6 \\
\hline
\end{tabular}




\section{Figure 1}

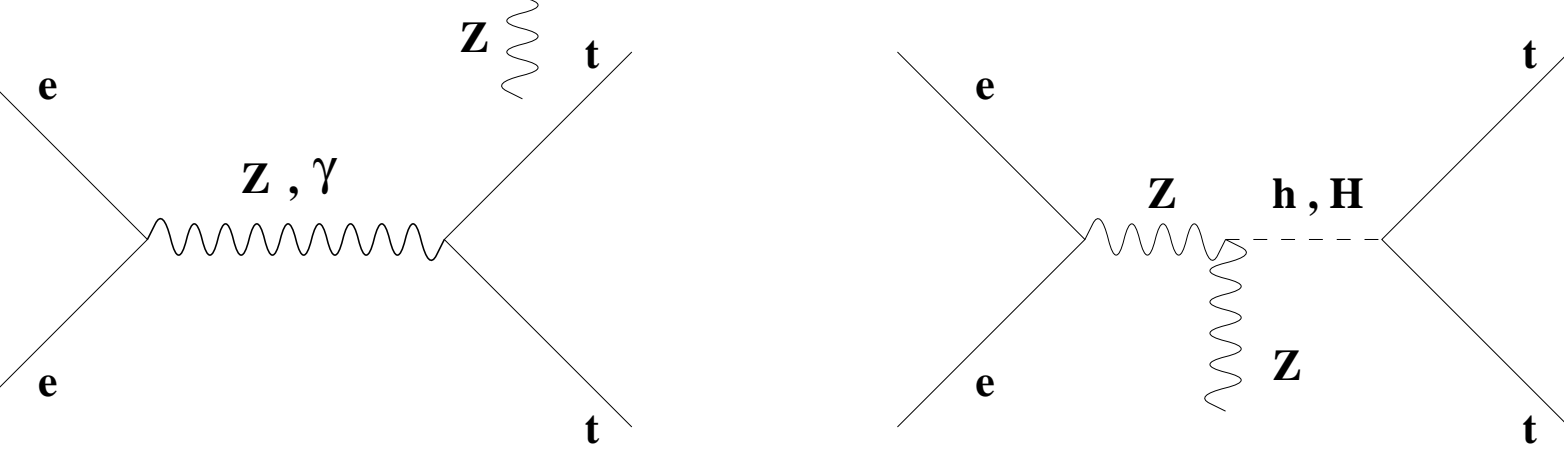

(a)

(b) 


\section{Figure 2}

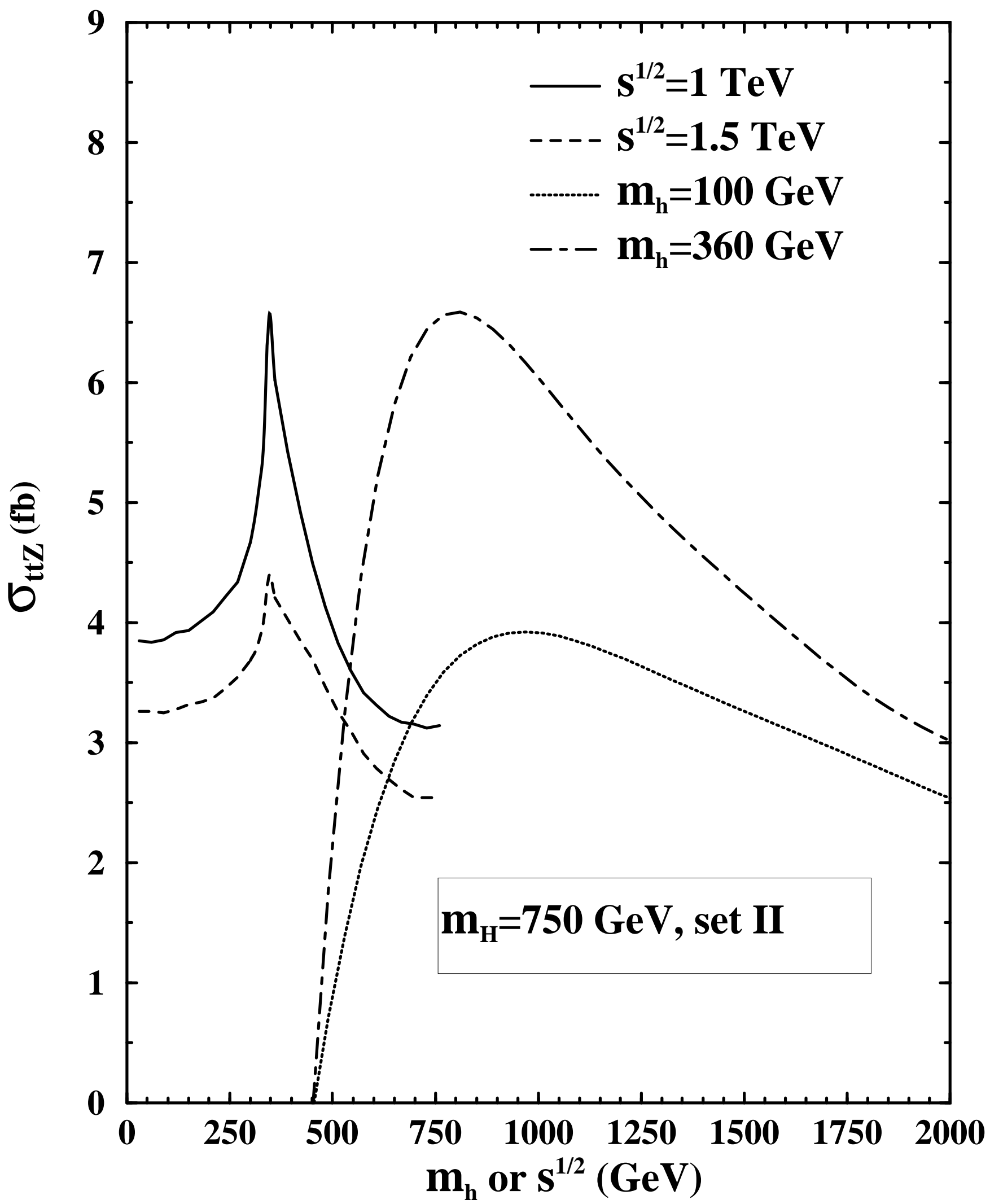




\section{Figure 3}

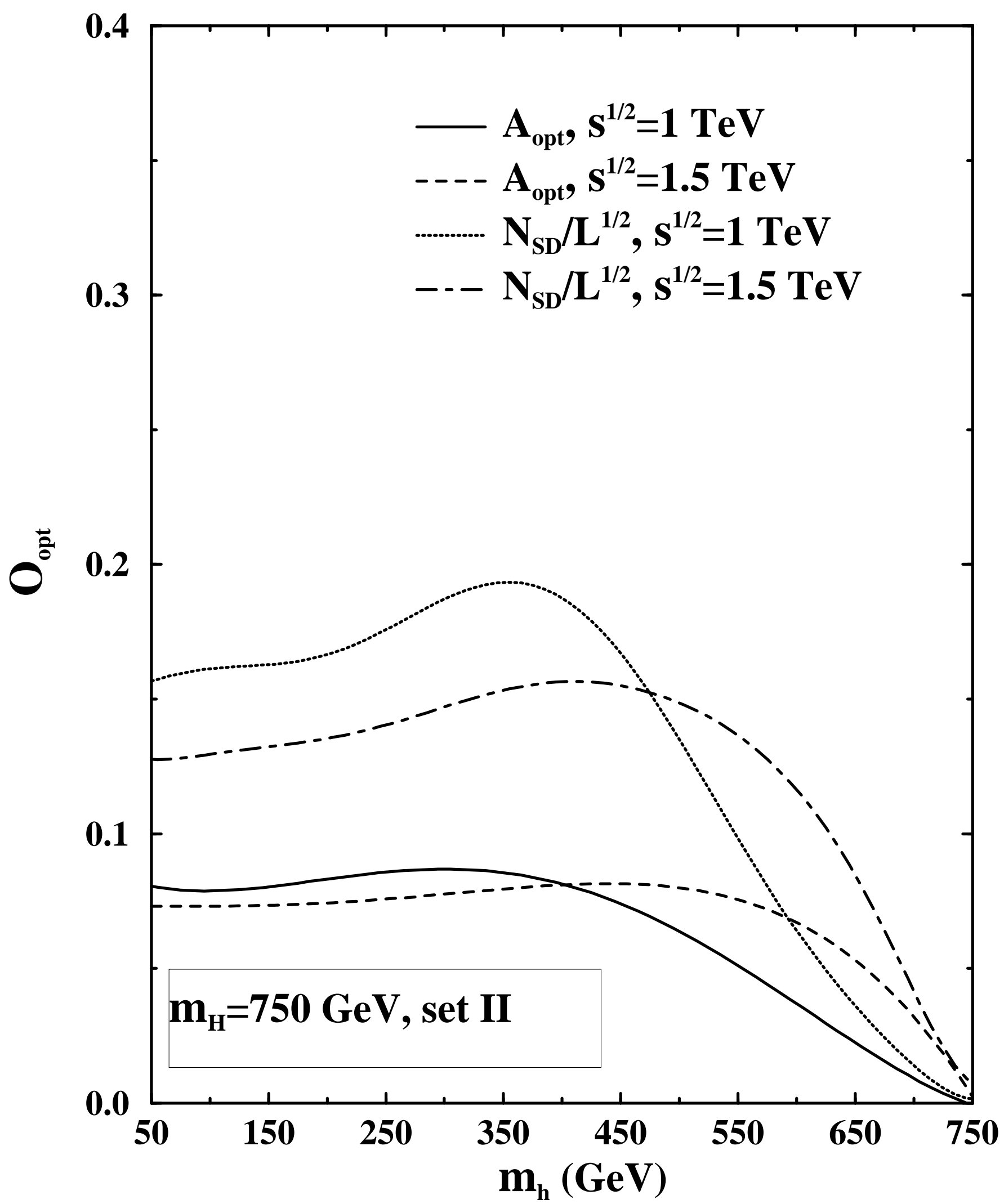

